René Tempelhoff mD Paul A. Modica MD, Edward L. Spitznagel Jr. PhD

\title{
Anticonvulsant therapy increases fentanyl requirements during anaesthesia for craniotomy
}

\begin{abstract}
This study was designed to determine whether patients receiving chronic anticonvulsant therapy demonstrate an altered requirement for fentanyl during anaesthesia. Sixty-one patients undergoing craniotomy were studied; 20 controls ( $M E D=0$ ) who had never received anticonvulsants and 41 epileptics in whom therapeutic plasma concentrations of either one $(M E D=1)$, two $(M E D=2)$, or three $(M E D=3)$ different anticonvulsants were documented. During anaesthesia with $60-70$ per cent $\mathrm{N}_{2} \mathrm{O}$ in $\mathrm{O}_{2}$ and 0.2 per cent isoflurane, a maintenance dose $(M D)$ of fentanyl was administered using a continuous variable-rate IV fentanyl infusion, supplemented by intermittent $50 \mu \mathrm{g} \mathrm{IV}$ boluses. In order to define the minimal dosage of fentanyl required, the $M D$ was titrated according to increases or decreases in the heart rate andlor mean arterial pressure exceeding 15 per cent of baseline ward values. A progressively higher fentanyl $M D$ was required in the epileptic patients (MED $=1-4.3 \pm 0.5 \mu \mathrm{g} \cdot \mathrm{kg}^{-1} \cdot \mathrm{hr}^{-1} ; M E D=2-5.4 \pm 0.6 ; M E D=$ $3-7.6 \pm 0.6)$ compared with the control $M D(M E D=0-2.6$ $\pm 0.5)(P<0.001)$. These findings indicate that there appears to be a dose-effect relationship between the number of anticonvulsants received and the maintenance dose of fentanyl required during balanced anaesthesia.
\end{abstract}

\section{Key words}

ANAESTHETICS, INTRAVENOUS: fentanyl; ANTICONVULSANTS: carbamazepine, phenytoin, primidone, valproic acid; COMPLICATION: epilepsy; PHARMACOLOGY: drug interactions - anticonvulsants, fentanyl.

From the Department of Anesthesiology, Division of Neuroanesthesia, Washington University School of Medicine, Box 8054, 660 S. Euclid Ave., St. Louis, MO 63110.

Address correspondence to: $\mathrm{Dr}$. R. Tempelhoff.

Presented in part at the 1988 Annual Meeting of the American Society of Anesthesiologists in San Francisco, California.
Le but de cette étude est d'observer les effets du traitement anticonvulsant de longue durée sur la dose de fentanyl requise pendant l'anesthésie. Soixante et un malades pour chirurgie intracranienne ont été etudiés de façon prospective: 20 patients controle $(M E D=0)$ qui n' ont jamais recus d'anticonvulsant et 41 épileptiques avec des niveaux plasmatiques thérapeutiques pour un $(M E D=1)$, deux $(M E D=2)$ ou trois $(M E D=3)$ anticonvulsants. La maintenance de l'anesthésie était assurée avec 70 pour cent $\mathrm{N}_{2} \mathrm{O}-\mathrm{O}_{2}, 0.2$ pour cent isoflurane et une perfusion intraveineuse a niveau variable de femanyl supplementée par des bolus intermittents de $50 \mu \mathrm{g}$. De manière a définir le dosage minimum de fentanyl requis (sans tenir compte du traitement anticonvulsant), la dose de maintenance de fentanyl ( $M D=$ perfusion + bolus) était augmentée ou diminuée selon les variations du rythme cardiaque et ou de la pression artérielle moyenne, pour les maintenir dans des limites n'éxcèdant pas \pm 15 pour cent de leurs valeurs preop obtenues dans le service. Une augmentation progressive de la MD de fentanyl a été requise chez les patients épileptiques des differents groupes $\left(M E D=1-4,3 \pm 0,5 \mu \mathrm{g} \cdot \mathrm{kg}^{-1} \cdot \mathrm{hr}^{-1} ; M E D=2-5,4 \pm 0,6\right.$ $M E D=3-7,6 \pm 0,6)$ quand comparé au groupe contrôle $(M E D=0-2,6 \pm 0.5)(P<0.001)$. Aucune différence significative entre le groupe contrôle et les patients épileptiques, ne fut notée concernant les differents critères d'émergence de l'anesthésie. Ces resultats semble indiquer qu'il existe une relation dose-effet entre le nombre d'anticonvulsants recus par les malades et la dose de maintenance de fentanyl requise pendant l'anesthésie générale "balancée".

Patients receiving one or more anticonvulsant medications, usually for the treatment of seizure disorders, are frequently encountered during anaesthetic practice. Recent studies have demonstrated a resistance to the neuromuscular blocking effects of both long- ${ }^{1,2}$ and intermediate ${ }^{3-5}$ acting nondepolarizing muscle relaxants in patients treated chronically with various anticonvulsants. The mechanism for the observed resistance is unclear. As anaesthetists regularly involved in the care of patients undergoing epilepsy surgery, we have often 
noticed that these patients also appeared to be resistant to the usual clinical doses of narcotics. ${ }^{6}$ Based on this clinical observation, we designed a study to determine whether or not patients receiving long-term anticonvulsant therapy demonstrated an altered requirement for fentanyl during anaesthesia.

\section{Methods}

Following approval by the Institutional Review Board of Washington University School of Medicine, 61 consenting patients between the ages of 16 to 60 years were studied. Patients with a history of renal, hepatic, metabolic, or cardio-pulmonary disease (including arterial hypertension) were excluded from the study, as were patients with known alcohol or narcotic abuse and patients demonstrating signs of increased intracranial pressure. The control group consisted of 20 otherwise healthy patients undergoing various craniotomies (e.g., tumour, arteriovenous malformation, cerebrospinal fluid leak) who had never received anticonvulsant therapy. The remaining 41 patients were epileptics undergoing craniotomy for seizure focus excision who had been treated for several years with various anticonvulsant regimens. The plasma concentration of each anticonvulsant received was in the therapeutic range the day prior to surgery. On the morning of surgery, anticonvulsant medications were reduced (usually in half) in most patients. All study patients were within 30 per cent of ideal body weight.

Each patient received premedication with ranitidine, $150 \mathrm{mg}$, and metoclopramide $10 \mathrm{mg}$, both given orally, 1-2 hrs before surgery. Upon arrival in the operating room and throughout anaesthesia, all patients were monitored with electrocardiogram (ECG), blood pressure (BP) cuff (Dinamap), pulse oximeter, and mass spectrometer. In all cases, an intra-arterial cannula was inserted for direct measurement of $\mathrm{BP}$ and arterial blood gas $(\mathrm{ABG})$ tensions. Tidal volume and respiratory rate were controlled to maintain end-tidal $\mathrm{CO}_{2}$ at $25 \mathrm{mmHg}$. Inspired gases were humidified to maintain oesophageal temperature above $35^{\circ} \mathrm{C}$. Blood loss, minimal in most cases, was closely monitored and isotonic IV fluids were administered in order to maintain euvolaemia.

The individuals responsible for anaesthetic administration (nurse anaesthetists familiar with neurosurgical procedures) were unaware of the purpose of the study or the possible association between anticonvulsant therapy and fentanyl requirement. Furthermore, the type, number and plasma concentrations of the anticonvulsant(s) were never discussed between the investigators and the nurse anaesthetist in charge of anaesthetic administration. These individuals were experienced in the technique of variable-rate narcotic infusion. However, they were not blinded to the amount or rate of fentanyl administration because they had to adhere to the anaesthetic protocol outlined in the following paragraphs.

Prior to induction of anaesthesia, incremental $50 \mu \mathrm{g}$ doses of fentanyl were given IV to each patient until he appeared to be sedated but still able to respond to simple commands. Anaesthesia was then induced with thiopentone, $4 \mathrm{mg} \cdot \mathrm{kg}^{-1}$, IV. Following moderate hyperventilation by mask with 100 per cent oxygen $\left(\mathrm{O}_{2}\right)$, tracheal intubation was facilitated with atracurium, $0.5 \mathrm{mg} \cdot \mathrm{kg}^{-1}$ IV. The induction was considered adequate if the HR and MAP immediately following intubation remained within 15 per cent of the patient's baseline ward values. Baseline ward HR and MAP were defined as the mean of the values obtained by nurses during the first $24 \mathrm{hr}$ after hospital admission. If the induction was inadequate (e.g., increase in HR and/or MAP exceeding 15 per cent of baseline), additional $50 \mu \mathrm{g}$ doses of IV fentanyl were given until the HR and MAP returned to within 15 per cent of baseline. The dose of fentanyl administered prior to and immediately following intubation was defined as the loading dose (LD) in $\mu \mathrm{g} \cdot \mathrm{kg}^{-1}$.

Anaesthesia was maintained during surgery with 60 70 per cent nitrous oxide $\left(\mathrm{N}_{2} \mathrm{O}\right)$ in $\mathrm{O}_{2}, 0.2$ per cent endtidal isoflurane, fentanyl infusion, and atracurium for muscle relaxation as indicated by ulnar nerve stimulation. The fentanyl infusion was started soon after induction at an initial maintenance infusion rate (MIR) of $1 \mu \mathrm{g} \cdot \mathrm{kg}^{-1}$. $\mathrm{hr}^{-1}$. Following surgical incision and until the start of closure, the MIR was considered adequate if the HR and MAP remained within \pm 15 per cent of baseline values. With time, whenever inadequate anaesthesia (e.g., increase in HR and/or MAP exceeding 15 per cent of baseline) was noted, incremental $50 \mu \mathrm{g}$ boluses of IV fentanyl were given and the MIR was increased by 0.5 $\mu \mathrm{g} \cdot \mathrm{kg}^{-1} \cdot \mathrm{hr}^{-1}$ until the HR and MAP again decreased to within 15 per cent of baseline. Conversely, the MIR was decreased in steps of $0.5 \mu \mathrm{g} \cdot \mathrm{kg}^{-1} \cdot \mathrm{hr}^{-1}$ (to a minimum MIR of $0.5 \mu \mathrm{g} \cdot \mathrm{kg}^{-1} \cdot \mathrm{hr}^{-1}$ ) if a decrease in HR and/or MAP exceeding 15 per cent of baseline occurred. The dose, boluses plus infusion, of fentanyl administered during maintenance of anaesthesia was defined as the maintenance dose (MD) in $\mu \mathrm{g} \cdot \mathrm{kg}^{-1} \cdot \mathrm{hr}^{-1}$. The LD and $\mathrm{MD}$ doses of fentanyl administered during each craniotomy were summated to give the total dose (TD) in $\mu \mathrm{g} \cdot \mathrm{kg}^{-1}$.

At the onset of surgical closure, isoflurane was discontinued and no additional boluses of fentanyl were administered. The fentanyl infusion rate was then progressively titrated downward with discontinuation at skin closure. Hypertension during emergence from anaesthesia (defined as a systolic BP > $160 \mathrm{mmHg}$ and/or a diastolic BP 
$>90$ ) was treated with conventional IV antihypertensive drugs (e.g., labetalol, hydralaxine) as required. After completion of closure, muscle relaxation was antagonized with neostigmine, $0.07 \mathrm{mg} \cdot \mathrm{kg}^{-1} \mathrm{IV}$, and glycopyrolate, $0.1 \mathrm{mg} \cdot \mathrm{kg}^{-1} \mathrm{IV}$, if required.

When the head dressing was completed, $\mathrm{N}_{2} \mathrm{O}$ was discontinued and 100 per cent $\mathrm{O}_{2}$ administered. The duration of anaesthesia was calculated as the time from the start of induction of anaesthesia until the discontinuation of $\mathrm{N}_{2} \mathrm{O}$. The time to awakening, min, was defined as the time from discontinuation of $\mathrm{N}_{2} \mathrm{O}$ until the patient was able to respond to simple commands and sustain an adequate respiratory pattern (e.g., negative inspiratory force (NIF) $\leq-20 \mathrm{~cm} \mathrm{H} \mathrm{H}_{2} \mathrm{O}$; end-tidal $\mathrm{CO}_{2} \leq 45$ torr; respiratory rate $>10$ per min; $\mathrm{O}_{2}$ saturation $\geq 98$ per cent). If the patient met these criteria, the trachea was extubated. If these criteria were not met within ten minutes, then naloxone, 0.2 to $0.4 \mu \mathrm{g} \cdot \mathrm{kg}^{-1} \mathrm{IV}$, was administered slowly and repeated once five minutes later if necessary. If the extubation criteria were still not met after two doses of naloxone, continuation of mechanical ventilation was planned until no longer required. Following extubation, each patient was observed for at least one hour in the recovery room, while breathing 35-40 per cent $\mathrm{O}_{2}$ via high-humidity mask. If felt to be clinically indicated, $A B G$ tensions were measured. All patients were discharged from the recovery room when they were awake and aware of their surroundings with stable vital signs.

After completion of data collection, the 61 study patients were retrospectively divided into the following four medication (MED) groups based on the number of anticonvulsants each was receiving.

$\mathrm{MED}=0:$ the 20 control patients who were not receiving anticonvulsant therapy.
$\mathrm{MED}=1: 16$ patients receiving carbamazepine

$\mathrm{MED}=2: 12$ patients receiving carbamazepine and either valproic acid or phenytoin.

$\mathrm{MED}=3: 13$ patients receiving carbamazepine, valproic acid and either phenytoin or primidone.

Data were analyzed as follows. Analysis of covariance (ANCOVA) with age and weight as covariables was performed to test the interaction between the four MED groups and the: (1) fentanyl LDs; (2) fentanyl MDs; (3) fentanyl TDs; (4) durations of anaesthesia; (5) awakening times; and (6) recovery room $A B G$ tensions. If the interaction was significant, multiple comparisons between MED groups were made using Bonferroni's correction in order to ensure overall protection. Fisher's exact test was performed to compare the control $(\mathrm{MED}=0)$ and epileptic $(M E D \geq 1=M E D=1+M E D=2+M E D=$ 3) patient groups with regard to the presence or absence of: (1) hypertension requiring therapy during emergence, and (2) respiratory depression requiring antagonism with naloxone during awakening. Values were considered significantly different if they achieved a $P<0.05$ or its Bonferroni corrected equivalent.

\section{Results}

The loading, maintenance and total doses of fentanyl required in each MED group, as well as the anaesthesia and awakening times are summarized in the Table. No significant differences in fentanyl LDs, durations of anaesthesia or awakening times were demonstrated between the four MED groups. However, the 41 patients (MED $\geq 1$ ) receiving long-term anticonvulsant therapy required a significantly higher fentanyl $M D$ and TD when compared with the 20 control $(\mathrm{MED}=0$ ) patients who were not treated with anticonvulsants $(P<0.001)$. Multiple comparison testing also revealed that the MDs

TABLE Loading $\left(\mu \mathrm{g} \cdot \mathrm{kg}^{-1}\right)$, maintenance $\left(\mu \mathrm{g} \cdot \mathrm{kg}^{-1} \cdot \mathrm{hr}^{-1}\right)$ and total $\left(\mu \mathrm{g} \cdot \mathrm{kg}^{-1}\right)$ doses of fentanyl with anaesthesia and awakening times (min) in the four anticonvulsant med groups $(n=61)$

\begin{tabular}{lccccc}
\hline \multicolumn{5}{c}{ Anticonvulsant medication groups } \\
\cline { 2 - 6 } & $M E D=0$ & $M E D=I$ & MED $=2$ & MED $=3$ & ANCOVA \\
\hline Number ( $n$ ) & 20 & 16 & 12 & 13 & \\
Loading dose (LD) & $6.8 \pm 0.9$ & $8.8 \pm 1.0$ & $8.6 \pm 1.2$ & $8.7 \pm 1.2$ & NS \\
Maintenance dose (MD) & $2.6 \pm 0.5$ & $4.3 \pm 0.5$ & $5.4 \pm 0.6 \dagger$ & $7.6 \pm 0.6 \dagger \ddagger$ & $*$ \\
Total dose (TD) & $22.9 \pm 4$ & $35.7 \pm 4$ & $42.9 \pm 5 \dagger$ & $55.4 \pm 4 \dagger \ddagger$ & $*$ \\
Duration of anaesthesia & $371 \pm 31$ & $375 \pm 35$ & $381 \pm 34$ & $369 \pm 30$ & NS \\
Awakening time & $5.9 \pm 0.8$ & $5.1 \pm 0.8$ & $5.6 \pm 1.0$ & $6.8 \pm 1.0$ & NS \\
\hline
\end{tabular}

All values are expressed as least square means $\pm \mathrm{SE}$.

NS $=$ not significant.

$* P<0.001$ for maintenance (MD) and total (TD) dose vs anticonvulsant medication (MED) groups.

†Significantly different from control (MED $=0$ ) group.

$¥$ \ignificantly different from $M E D=1$ group. 


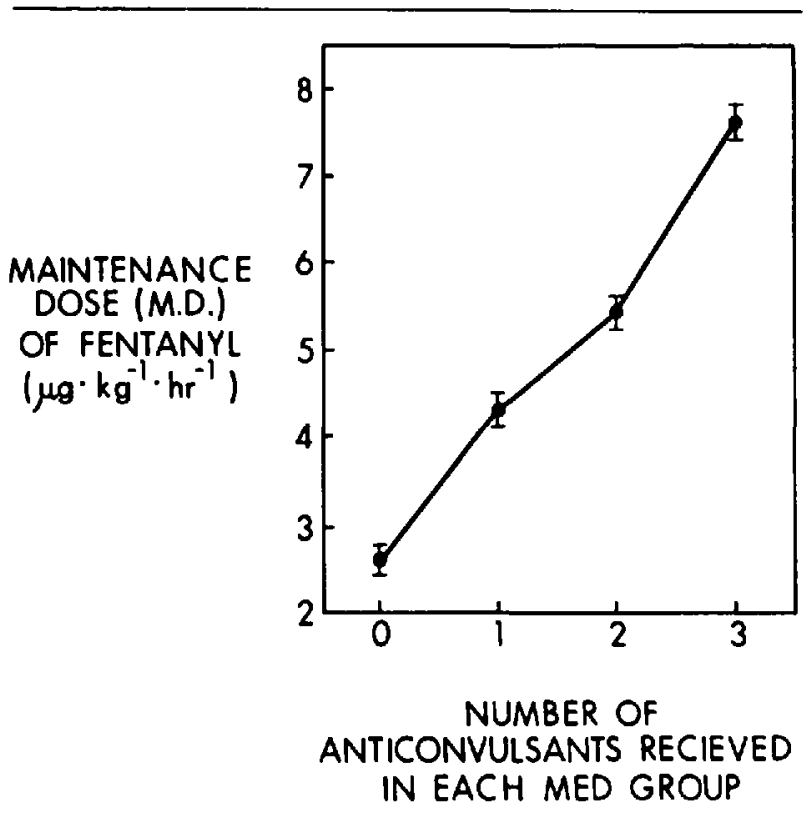

FIGURE Lincar dose-effect relationship between the number of anticonvulsants received in each medication (MED) group and the maintenance dose (MD) of fentanyl required. The plasma level of each anticonvulsant received was in the therapeutic range prior to surgery. Values are expressed as least square means \pm SE $(P<0.001$ by analysis of covariance).

and TDs in the MED $=2$ and MED $=3$ groups were significantly greater when each was compared with the control (MED =0) MD. In addition, the MD and TD in MED $=3$ was significantly higher when compared with the MD and TD in MED = 1 . Overall, as shown in the Figure, there was no evidence for anything beyond a linear dose-effect relationship between the number of anticonvulsants with therapeutic plasma levels received in each MED group and the MD of fentanyl required. This finding for MD also led to a similar dose-effect relationship for TD (MD + LD) because LD was not significantly different between the four MED groups (see Table).

Hypertension requiring therapy during emergence occurred in 16.4 per cent of the 61 study patients. Within each of the four MED groups the distribution of emergence hypertension was: 25 per cent in MED $=0 ; 12.5$ per cent in $\mathrm{MED}=1 ; 8.3$ per cent in $\mathrm{MED}=2$; and 15.4 per cent in $\mathrm{MED}=3$. The incidence of hypertension requiring therapy during emergence did not differ significantly between the 20 control $(M E D=0)$ and 41 epileptic (MED $\geq 1)$ patients $(P=0.18)$.

Respiratory depression requiring antagonism with naloxone during awakening occurred in 9.8 per cent of the 61 study patients. Within each of the four MED groups, the distribution of respiratory depression was: 10 per cent in $\mathrm{MED}=0 ; 12.5$ per cent in $\mathrm{MED}=1 ; 8.3$ per cent in $\mathrm{MED}=2$; and 7.7 per cent in $\mathrm{MED}=3$. The incidence of respiratory depression requiring naloxone therapy during awakening did not differ significantly between the 20 control $(M E D=0)$ and 41 epileptic $(M E D \geq 1)$ patients $(P=0.65)$. Antagonism by naloxone was successful in all six cases and no study patient required tracheal intubation after leaving the operating room.

Recovery room arterial blood gas tensions were measured in 32 study patients. No significant differences in postoperative $\mathrm{pH}, \mathrm{PO}_{2}$, or $\mathrm{PCO}_{2}$ existed among the four MED groups.

\section{Discussion}

This study was designed to define the minimal dosage of fentanyl required during anaesthesia using a balanced technique in patients undergoing craniotomy, regardless of their anticonvulsant history. Our results demonstrated that epileptic patients treated chronically with one or more anticonvulsants have higher fentanyl requirements for maintenance of anaesthesia. Furthermore, there seems to be a dose-effect relationship between the number of anticonvulsants received and the maintenance dose (MD) of fentanyl required during balanced anaesthesia. The scale of the $x$-axis in the Figure does not imply that a patient treated with two different types of anticonvulsants is receiving twice the level of anti-seizure effect as someone receiving only one drug. It was merely used to indicate the difference in fentanyl requirements between the three MED groups. It is impossible to quantify the level of additional anti-seizure effect in patients treated with two or more different types of anticonvulsants when compared to someone receiving only one drug.

Alterations in fentanyl dosage requirements in other patient populations have been previously reported. A history of smoking, alcohol, or caffeine consumption increases the requirement for fentanyl. ${ }^{7}$ When compared with patients with normal left ventricular function, ${ }^{8}$ smaller doses of fentanyl are required for unresponsiveness in patients with depressed ventricular function. ${ }^{9}$ Overall, the pharmacokinetics for fentanyl are remarkably inconstant even in healthy volunteers. ${ }^{10}$

Interactions between various anticonvulsants and other drugs used in anaesthesia have been demonstrated previously. Chronic therapy with either phenytoin ${ }^{1,3}$ or carbamazepine $^{2,4}$ alone has been shown to accelerate recovery from the neuromuscular blocking effects of both long-acting muscle relaxants and vecuronium. In epileptics treated with multiple anticonvulsant therapy, a similar resistance to atracurium ${ }^{5}$ was found; however, this drug interaction was not demonstrated by other investigators in patients receiving single anticonvulsant therapy. ${ }^{3,4}$ A 25 per cent increase in the hepatic clearance of meperidine was reported in healthy volunteers pretreated with either phenobarbital $^{11}$ or phenytoin ${ }^{12}$ for 10-14 days. Experi- 
mentally, chronic ingestion of phenobarbital produced hepatic necrosis in rats exposed to one per cent halothane under hypoxaemic conditions. ${ }^{13}$

Various pharmacokinetic and pharmacodynamic mechanisms could explain the observed resistance to fentanyl seen with anticonvulsants in this study. Chronic administration of phenobarbitone ${ }^{13}$ and phenytoin ${ }^{14}$ has been shown to accelerate drug biotransformation due to stimulation of microsomal enzyme system activity. Since the clearance of fentanyl is primarily hepatic, ${ }^{10}$ the finding in our epilepitc patients of a higher MD without a difference in the LD of fentanyl (compared with control) could imply enhanced fentanyl metabolism due to hepatic enzyme induction.

Although this appears to be an attractive hypothesis for the drug interaction between fentanyl and anticonvulsants, alterations in the central nervous system could be also involved. In rats, electrical recordings at single cells in the locus coeruleus (one of the most dense clusters of opiate receptors in the brain) revealed that low concentrations of opiates inhibit firing of these neurons. ${ }^{15}$ Conversely, carbamazepine produces a dose-dependent activation of these neurons, ${ }^{16}$ a finding similar to that shown for naloxone in rats chronically treated with morphine. ${ }^{17}$ In view of these findings, changes in the state of opiate receptors induced by chronic anticonvulsant exposure may be operative.

Due to the obvious differences in surgical indications, it was not possible to conduct this study in a true double-blind fashion, because the study patients undergoing seizure surgery were readily distinguishable from the control group undergoing craniotomy for other neurological disorders. However, in order to minimize bias during fentanyl administration, the nurse anaesthetists responsible for drug administration were unaware of the purpose of this study or the possible association between anticonvulsant therapy and fentanyl requirements. They were instructed to administer fentanyl in a variable-rate fashion according to a strict protocol in order to provide an adequate level of anaesthesia using the minimal dosage of narcotic required. Thus, although the medical records were available, it is unlikely that they were biased in their approach to fentanyl administration.

Previous investigations have used variable-rate infusion techniques to determine the minimal narcotic dosage required during anaesthesia. In these studies, haemodynamic (changes in HR and/or MAP), somatic (e.g., swallowing, eye opening, bodily movements) and sympathetic (e.g., tearing, mydriasis, sweating) criteria were used to dictate the need for additional narcotic, as there was no single response that was consistently exhibited as a clinical sign of light anaesthesia. ${ }^{18-20}$ In contrast to these earlier studies, in our control and study patients, most of the somatic and sympathetic signs of inadequate anaesthesia were obfuscated by various factors inherent in the anaesthetic management for craniotomy, such as patient positioning, surgical draping and muscle relaxation. Thus, we were restricted to the use of haemodynamic signs of light anaesthesia as the basis upon which to adjust the rate of fentanyl administration, to define the minimal dosage required in each MED group. Despite this limitation, our variable-rate infusion model appeared to be well-suited for studying the fentanyl-anticonvulsant drug interaction, inasmuch as we were unable to demonstrate any differences between the control and epileptic patients with regard to: (1) emergence hypertension; (2) awakening times; (3) respiratory depression requiring naloxone antagonism; and (4) recovery room ABG tensions. The similarity of these emergence and recovery characteristics among all patients studied further supports our finding that the average fentanyl MD required for an epileptic patient was approximately 1.5 - to 3 -fold higher than in patients not receiving anticonvulsant therapy.

In an effort to provide better protection against intraoperative awareness, we chose to supplement our nitrous oxide-narcotic-relaxant technique with 0.2 per cent endtidal isoflurane. The effect of this small amount of isoflurane is probably not an important issue in this study, as all four MED groups received this agent in equal concentrations. Furthermore, the high doses (22.9 \pm 4 to $55.4 \pm 4 \mu \mathrm{g} \cdot \mathrm{kg}^{-1}$ total) of fentanyl administered and the rapid awakening times $(5.1 \pm 0.8$ to $6.8 \pm 1.0 \mathrm{~min})$ suggest that the contribution of this low dose of isoflurane to the overall anaesthetic was small.

Conservative estimates indicate that about 60,000 individuals in the United States have focal epilepsy that would benefit from surgery. ${ }^{6}$ Only a few hundred undergo operations for epilepsy annually, but a great number of them will require surgery during their lifetimes for the treatment of other illnesses. This study demonstrates that patients chronically treated with anticonvulsants may also exhibit a higher requirment for fentanyl. The mechanisms for the observed resistance are unclear. Further pharmacokinetic and neurobiological studies will be necessary to understand these phenomena.

\section{References}

1 Ornstein E, Matteo RS, Young WL, Diaz J. Resistance to metocurine-induced neuromuscular blockade in patients receiving phenytoin. Anesthesiology 1985; 63: 294-8.

2 Roth $S$, Ebrahim ZY. Resistance to pancuronium in patients receiving carbamazepine. Anesthesiology 1987; 66: 691-3.

3 Ornstein E, Matteo RS, Schwartz AE, Silverberg PA Young $W L$, Diaz $J$. The effect of phenytoin on the 
magnitude and duration of neuromuscular block following atracurium or vecuronium. Anesthesiology 1987; 67: 191-6.

4 Ebrahim Z, Bulkley R, Roth S. Carbamazcpine therapy and neuromuscular blockade with atracurium or vecuronium. Anesth Analg 1988; 67: S55.

5 Modica $P$, Tempelhoff $R$. Effect of chronic anticonvulsant therapy on recovery from atracurium. Anesth Analg $1989 ; 68 ; \mathrm{S} 198$.

6 Goldring S, Gregorie EM, Tempelhoff R. Neurosurgery. In: Rob and Smith's Operative Surgery, 4th ed., London, Butterworths, 1989.

7 Stanley $T H$, de Lange $S$. The influence of patient habits on dosage requirements during high dose fentanyl anesthesia. Can Anaesth Soc J 1984; 31: 368-76.

8 Stanley $T H$, Philbin DM, Coggins $C H$. Fentanyl-oxygen anesthesia for coronary artery surgery: cardiovascular and antidiuretic hormone responses. Can Anaesth Soc J 1979; 26: 168-72.

9 Stanley $T H$, Webster $L R$. Anesthetic requirements and cardiovascular effects of fentanyl-oxygen and fentanyldiazepam-oxygen anesthesia in man. Anesth Analg 1978; 57: 411-6.

10 Mather LE. Clinical pharmacokinetics of fentanyl and its new derivatives. Clin Pharmacokinet 1983; 8: 422-46.

11 Stambaugh JE, Wainer IW, Schwartz I. The effect of phenobarbital on the metabolism of meperidine in normal volunteers. J Clin Pharmacol 1978; 18: 482-90.

12 Pond SM, Kretschzmer KM. Effect of phenytoin on meperidine clearance and normeperidine formation. Clin Pharmacol Ther 1981; 30: 680-6.

13 McClain GE, Sipes IG, Brown BR. An animal model of halothane hepatotoxicity: roles of enzyme induction and hypoxia. Anesthesiology 1979; 51: 321-6.

14 Luoma PV. Sotaniemi EA, Arranto AJ. Serum LDL cholesterol, the LDL/HDL cholesterol ratio and liver microsomal enzyme induction evaluated by antipyrine kinetics. Scand J Clin Lab Invest 1983; 43: 671-5.

15 Bird SJ, Kuhar MJ. Iontophorectic application of opiates to the locus coeruleus. Brain Res 1977; 122: 523-33.

16 Olpe HR, Jones RSG. The action of anticonvulsant drugs on the firing of locus coeruleus neurons: selective, activating effect of carbamazepine. Eur J Pharmacol 1983; 91: $107-10$.
17 Aghajanian GK. Tolerance of locus coeruleus neurons to morphine and suppression of withdrawal response by clonidine. Nature 1978; 276: 86-8.

18 Ausems ME, Hug CC Jr, de Lange S. Variable rate infusion of alfentanil as a supplement to nitrous oxide anesthesia for general surgery. Anesth Analg 1983; 62 . 982-6.

19 White $P F$. Use of continuous infusion versus intermittent bolus administration of fentanyl or ketamine during outpatient anesthcsia. Anesthesiology 1983: 59: 294-300.

20 Ausems ME, Hug CC Jr, Stanski DR, Burm AGL. Plasma concentrations of alfentanil required to supplement nitrous oxide anesthesia for general surgery. Anesthesiology $1986 ; 65: 362-73$. 